\title{
The intraday variations of polarization vector direction in radio source S5 $0716+714$
}

\author{
Elena S. Shablovinskaya, ${ }^{1 \star}$ Viktor L. Afanasiev ${ }^{1}$ \\ ${ }^{1}$ Special Astrophysical Observatory of Russian Academy of Science, Nizhnii Arkhyz, 369167 Russia
}

Accepted XXX. Received YYY; in original form ZZZ

\begin{abstract}
The bright radio source S5 0716+714, that is usually classified as BL Lac object, is one of the most intensively studied. S5 $0716+714$ demonstrates extremely peculiar properties such as the shortest time-scale of optical and polarimetric variations observed in blazars. In given paper, the results of 9-hour polarimetric monitoring of S5 0716+714 with the $\sim 70$-second resolution carried out by the 6-m telescope BTA of SAO RAS, are presented. The observation data analysis reveals the variability both in total and polarized light on the 1-1.5-hour time-scale. Since polarization is generated by plasma motion in the magnetic field, the variations of polarization vector are bounded with the magnetic field configuration and the average time-scales specify the size of the emitting region, unresolved directly. We suggest a processed numerically model of the polarization in the jet with the precessing helical magnetic field. Fitting the model discovers that observed short-term variations with the complicated trajectory of polarization vector could be induced by the magnetic field precession with the period $T \approx 15$ days.
\end{abstract}

Key words: BL Lacertae objects: general - BL Lacertae objects: individual: S5 $0716+714$ - polarization - galaxies: jets

\section{INTRODUCTION}

The object S5 $0716+714$ (R.A.: $07^{h} 21^{m} 53^{s} .4$, Dec.: $\left.+71^{\circ} 20^{\prime} 36^{\prime \prime}\right)$ is a bright radio source with extraordinary variable brightness. According to Biermann et al. (1981), S5 0716+714 was classified as BL Lac object due to specific features common to the class: flat power-law spectrum ( $\alpha \geq-0.5, S_{v} \propto v^{\alpha}$ in radio band), high and variable optical polarization degree and significant variability in all wavebands. However, some authors mention fluctuations of intensity shown by S5 $0716+714$ - up to 5 mag in flares (Larionov et al. 2013) and up to $0.5 \mathrm{mag}$ within the night - are irrelevant to the sample of BL Lac objects.

Though the investigation of S5 $0716+714$ in all wavebands has been conducted for over 25 years, the object redshift is still an open issue. There is no sign of host-galaxy neither by direct photometric nor by spectral observations: there are no spectral features detected at the $0.3 \%$ level in optical band (Nilsson et al. 2008). The HST survey of BL Lacertae objects (Urry et al. 2000) had revealed no evidence of the host existence and therefore the severe restriction to the maximum host-galaxy brightness was given $-m>20.0$ mag that corresponded to the redshift $z>0.5$. However, the most authors accepts $z=0.1 \div 0.5$, obtained by ground-

^ E-mail: e.shablie@yandex.com based and still not concerted observations. The problem of S5 $0716+714$ redshift presently is not solved, and hence the extragalactic origin is not granted.

The S5 $0716+714$ brightness is variable across all the spectrum from radio - on the time-scale of days (Liu et al. 2017), 8 - 16 hours (Gorshkov et al. 2011a,b) - to $\gamma-15$ min (Biermann et al. 1981). According to the general model (Gabuzda 2013), the optical radiation of blazar is produced in the unresolved region of the jet at the distance $\lesssim 10^{-2} \mathrm{pc}$ from the centre (so-called optical jet), and the investigation of the regions near the central black hole is essential for such issues as jet formation and variability origin. Inside the jet, relativistic plasma radiates due to the motion in the ordered magnetic field producing non-thermal (synchrotron) polarized emission. The direction of polarization is determined by charged particles oscillations and so by their direction in the magnetic field. Therefore, the change of the polarization vector is a pointer of the field configuration.

At the optical jet scale plasma moves rapidly in a compact region that leads to polarization vector variability on short time-scales (intraday variability). The rapid variability both in total and polarized light was discovered: in (Impey et al. 2000) the significant polarization changes within less than 2 hours were pointed out; the similar result was obtained in (Amirkhanyan 2006) - within 3 hours of the observation set polarization degree noticeably changed with 


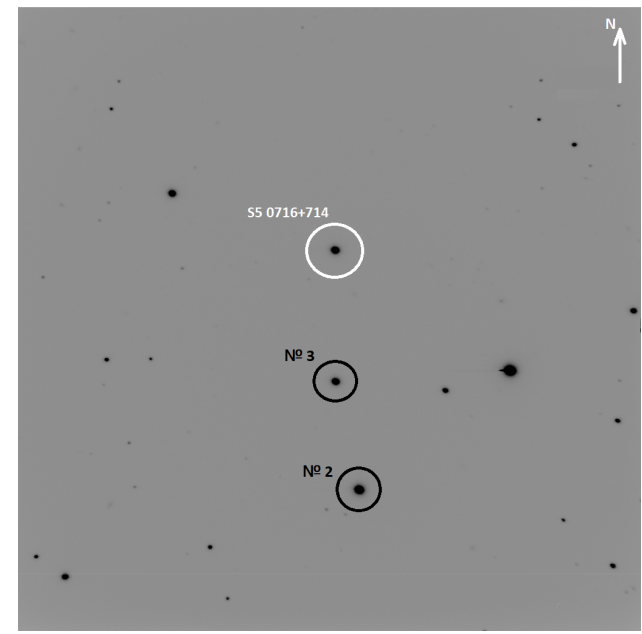

Figure 1. The finding chart of S5 $0716+714\left(\mathrm{FoV}-6.5^{\prime} \times 6.5^{\prime}\right)$.

the amplitude of $6 \%$. More recent papers (e.g. Zhang et al. 2018) revealed the intraday variability as well.

In the given work, the results of the rapid variability investigation in total and polarized light of S5 $0716+714$ are presented. The obtained observational data are interpreted within the model of the precessing helical magnetic field. Finally, the constructed numerical model qualitatively explains our observations and other authors' data.

\section{OBSERVATIONS}

On February 2018 our group conducted a polarimetric monitoring of S5 $0716+714$ to reveal intraday variability of flux and polarization. The observations were carried out with the SCORPIO focal reducer (Afanasiev \& Amirkhanyan 2014) at the primary focus of the 6-m BTA telescope of the Special Astrophysical Observatory of the Russian Academy of Sciences (SAO RAS) for 9 hours. We obtained 460 exposures each of $60 \mathrm{sec}$ in the g-SDSS filter, interrupted by approximately $10 \mathrm{sec}$ gaps.

A double Wollaston prism (Geyer et al. 1996; Oliva 1996) was used as a polarization analyser. The key benefit of observations with double Wollaston prism is that measurements in mutually perpendicular directions $0^{\circ}, 90^{\circ}$ and $45^{\circ}, 135^{\circ}$ are obtained jointly: the frame consists of four different images of the exit pupil, so the registration of the linear polarization parameters - the Stokes parameters $Q$ and $U-$ is simultaneous. This is particularly important for unstable atmospheric conditions: as the parameters of the radiative transfer in the atmosphere vary, scintillations and depolarization coefficients variations occur. Concerning the observations in $\mathrm{SAO}$, the main reason of depolarization is non-selective aerosol light scattering, so polarization changes up to several percents on time-scales of 10-30 msec.

The analyser was rotated to keep in the field of view not only S5 $0716+714$ but also two stars close to the object - in Fig. 1 №3 (R.A.: $07^{h} 21^{m} 54^{s}$.36, DEC.: $+71^{\circ} 19^{\prime} 20^{\prime \prime} .92, m_{B}=$ 14.15) and №2 (R.A.: $07^{h} 21^{m} 52^{s} .33$, DEC.: $+71^{\circ} 18^{\prime} 17^{\prime \prime} .71$, $\left.m_{B}=13.04\right)$. The first star was used as both a photometric and zero polarization standard (Amirkhanyan 2006); the star №2 was used to control accuracy. Then simultaneous ob-

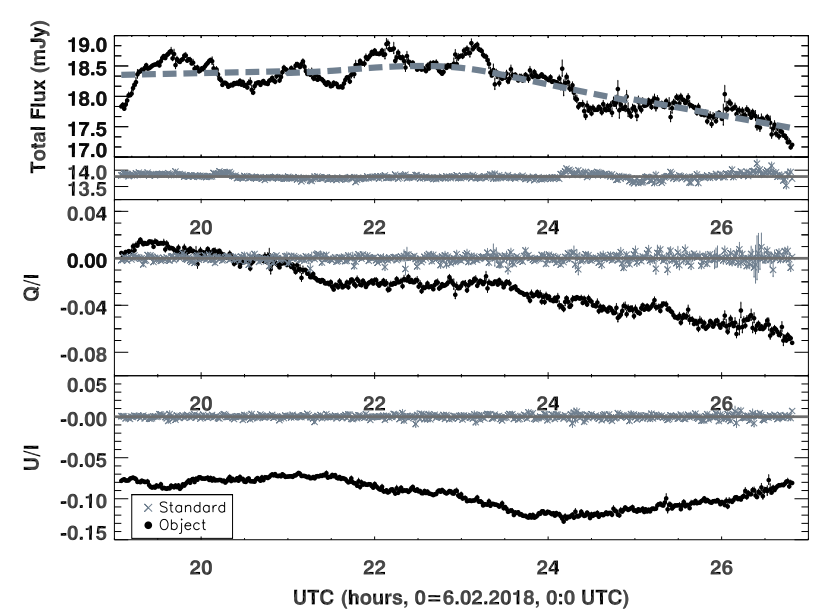

Figure 2. The variations of the total flux and the Stokes parameters $Q$ and $U$ within the night. The observations were started at 19:08 on 2nd February 2018 (UTC).

servations of the object and the standard admit to correct unsteady atmospheric absorption and, moreover, to minimize depolarization. The normalized Stokes parameters $Q$ and $U$ could be derived from the relations:

$$
\frac{Q}{I}=\frac{I_{0}-I_{90} D_{Q}}{I_{0}+I_{90} D_{Q}}, \frac{U}{I}=\frac{I_{45}-I_{135} D_{U}}{I_{45}+I_{135} D_{U}}
$$

where $D_{Q}$ and $D_{U}$ are coefficients of polarization channel transmission, calculated from the deviation of the independently measured standard star. $D_{Q}$ and $D_{U}$ are weakly timedependent, so considered to be constant: $D_{Q}=1.036 \pm 0.015$ and $D_{U}=0.985 \pm 0.015$. The total flux is defined:

$$
I=I_{0}+I_{90} D_{Q}+I_{45}+I_{135} D_{U}
$$

The proposed measurement technique provides the photometry accuracy above 0.005 mag and the polarimetry accuracy up to $0.1 \%$ on average. The results of our observations could be found in Fig. 2.

\subsection{The flux variations}

The variations of S5 $0716+714$ total flux are presented in the upper panel of Fig. 2 with black dots. The standard star flux is shown with grey crosses for comparison. The standard deviation of the flux is $\sigma=0.079$. The zenith distance of the object was changing from $27^{\circ} .6$ to $55^{\circ} .2$. While the comparison star has been observed at the same height, the effects of changing atmospheric absorption cancel out.

Also, throughout the night the total flux demonstrates the slight slope - the trend, and the quasi-periodic variations, not detected for the standard star. In Fig. 2 the trend is shown with a grey dash line. To investigate the periodicity of the source flux variations with the subtracted trend the wavelet analysis is applied to follow the dynamics of the harmonic component. The magnitude of the wavelet transform is presented in Fig. 3, where the areas of $0.7 \sigma, 1 \sigma$, $1.5 \sigma$ and $2 \sigma$ (or $95 \%$ ) deviation are countered. Evidently, the variation period is changing from 65 to $85 \mathrm{~min}$ within the night. Median wavelet magnitude profile (Fig. 4) reveals the average period $-\sim 77 \pm 10 \mathrm{~min}$. 


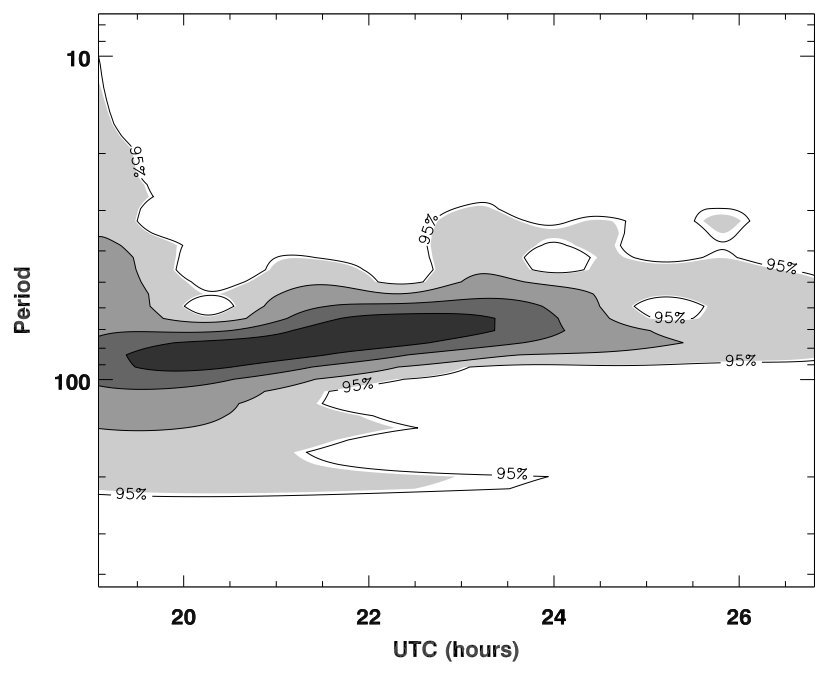

Figure 3. The magnitude of the wavelet transform.

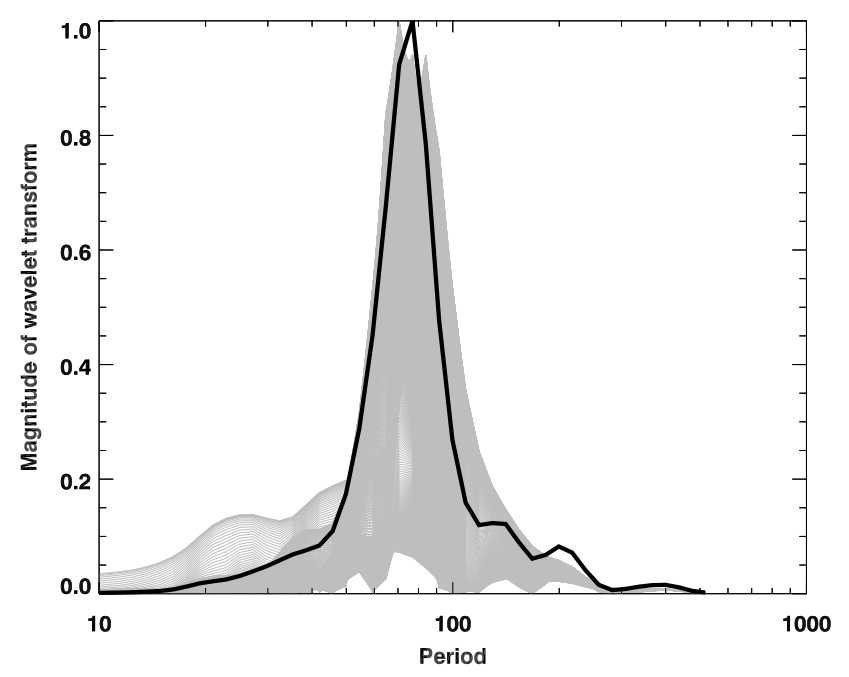

Figure 4. The wavelet transform profiles (grey) and their median value (black).

\subsection{The polarization variations}

The variations of linear polarization - the normalized Stokes parameters $Q$ and $U$ - are shown in the middle and bottom panels of Fig. 2, respectively. Similarly to the flux, the S5 $0716+714$ polarimetric data are presented with black dots, the standard star data - with grey crosses. The standard deviations are $\sigma_{Q}=0.0038$ and $\sigma_{U}=0.0023$. Evidently, within the night, the polarization has significantly changed by as much as $7 \%$, yet more substantial inferences could not be done. As the vector quantity, the polarization variations are performed on the $Q U$-diagram as on the most convenient pictorial rendition. Fig. 5 demonstrates the motion of the polarization vector during the observation set; the colours correspond to time. Moving along some complicated path, the vector switches the direction of the motion with the average time $\sim 1.5-3$ hours. It should be noticed that polarization

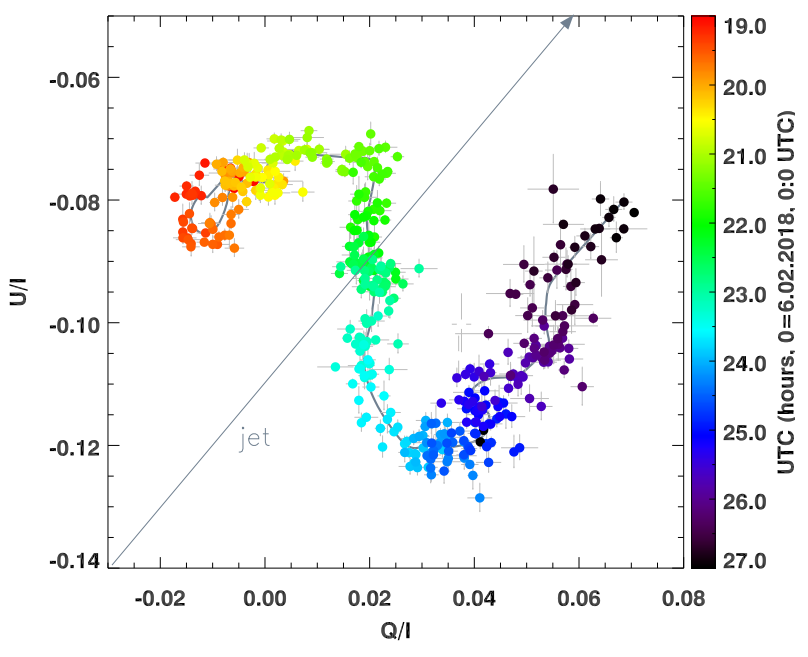

Figure 5. The variations of normalized Stokes parameters $Q$ and $U$ within the night on the $Q U$-diagram.

variations time-scale is of the order for the total flux ones. The physical interpretation would be given in Section 4 .

To examine the polarization vector variability together with the jet location the optical jet is assumed to coincide with the radio jet at the position angle $\sim 25^{\circ}$ (Rastorgueva et al. 2011). As on the $Q U$-plane the angles double, the jet position is $50^{\circ}$ (see Fig. 5). Then it occurs that the fluctuations of the polarization vector and, consequentially, the charged particles motion are perpendicular to the jet direction.

An important point is that in several papers (e.g. Impey et al. 2000) the variations of flux and polarization on the time-scales of 1-10 min are asserted found out. Actually, it could be suggested from the Fig. 2 and 5, yet comparing with the standard one could reveal this kind of scintillations is the atmospheric noises, not connected with the source.

\section{MODEL OF POLARIZATION IN JET}

The application of magnetohydrodynamic (MHD) models to astrophysical jets (e.g. Meier et al. 2001) reveals that inside the flow the instabilities such as kink-instability could develop that leads to a helical structure formation in plasma (e.g. Zhang et al. 2017). The observations of the near active galaxies such as M87 (Capetti et al. 1997) confirm this theoretical model. In the given section, we would like to suggest our geometrical model of polarization in the jet with the helical structure of the magnetic field.

To describe the jet geometry a reference system $(x, y, z)$ is applied, for which $x$-direction corresponds to the jet projection to the celestial plane for the observer, $z$ is directed along the jet, and $y$ forms an orthogonal basis with $x$ and $z$ (Fig. 6). The plasma motion in the helical magnetic field in the conical jet is described in a cylindrical frame $(\rho, \psi, z)$ where $\rho$ is the distance from the point to the applicate axis, $\psi$ is the azimuth and $z$ is the distance along the applicate coincided with $z$-axis in $(x, y, z), \psi$ is counted from $x$-direction. Assuming angular momentum $L$, kinematic energy $E_{k i n}$ and half-opening angle $\theta$ to be constant, the equations of motion 


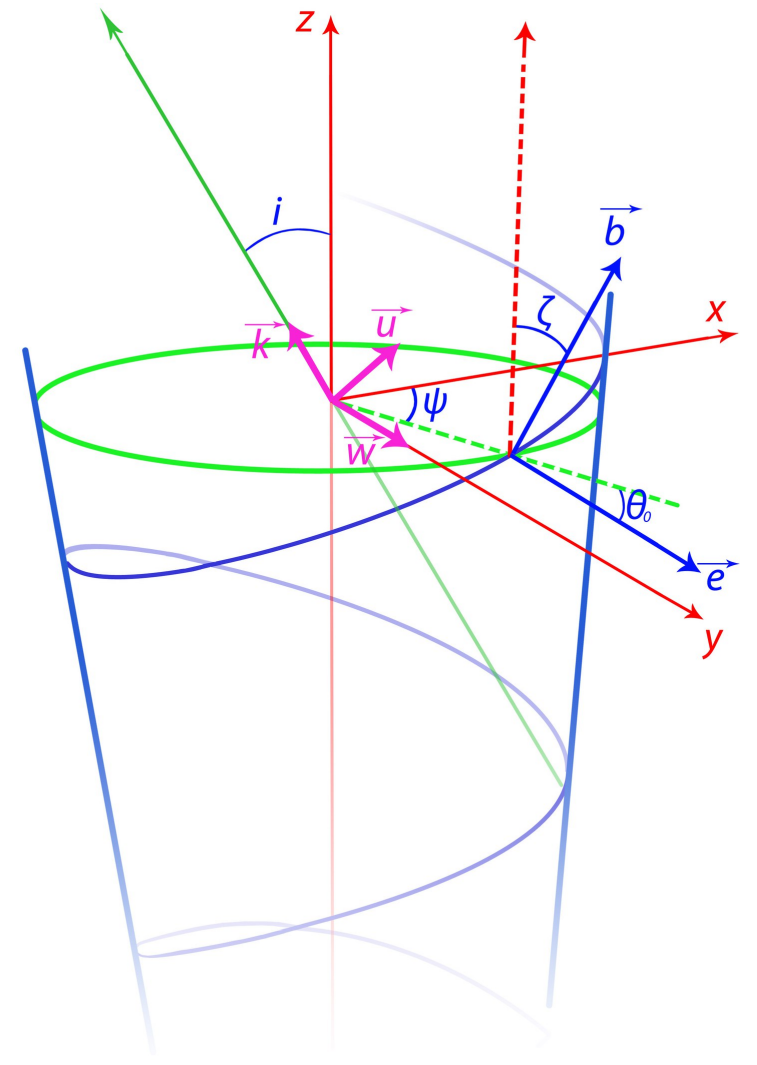

Figure 6. The reference systems established to describe charged particle motion in jet.

become (Steffan et al. 1995; Li et al. 2018):

$$
\begin{gathered}
\rho=f \sqrt{1+\left(\frac{a t+b}{f}\right)^{2}}, \quad \dot{\rho}=\frac{a}{\rho}(a t+b) \\
\psi=\frac{\left[\operatorname{arctg}\left(\frac{a t+b}{f}\right)-\operatorname{arctg}\left(\frac{b}{f}\right)\right]}{\sin \theta}, \quad \dot{\psi}=\frac{a f}{\rho^{2} \sin \theta} \\
z=\frac{\rho-\rho_{0}}{\operatorname{tg} \theta}, \dot{z}=\frac{\dot{\rho}}{\operatorname{tg} \theta},
\end{gathered}
$$

where the next constants are used:

$$
a=\beta \sin \theta, \quad b=\sqrt{\rho_{0}^{2}-f^{2}}, \quad f=\frac{j}{\beta},
$$

where $\beta=v / c$ is the physical speed of a jet component in units of the speed of light $c, \rho_{0}$ is the cylindrical distance, $j=\frac{L}{E_{\text {kin }}} c$ is angular momentum in units of distance. Time $t$ is also expressed in distance units, i.e. $t \equiv t c$.

Fig. 6 displays that instantaneous velocity of the charged particle would be characterized by the vector $\mathbf{b}$ along the particle direction and the vector e perpendicular to the cone side and helical trajectory as well. e indicates electric vector, indeed, therefore at every moment the observed direction of the polarization vector is determined by the instantaneous position of the vector e projected to the celestial sphere assigned by $(u, w)$ reference frame. The vector $\mathbf{k}$ and $(u, w)$ also form the basis, $\mathbf{k}$ is pointed to the observer at the angle $i$ to the jet axis.
Then the direction of the electric vector in the observer frame is determined by polarization angle $\chi$ :

$$
\tan \chi=\frac{e_{u}}{e_{w}}=\frac{\cos \psi \cos i-\tan \theta_{0} \sin i}{\sin \psi},
$$

where $\theta_{0}$ is the angle between e and the plane of the instantaneous particle position, or $(x, y)$ plane. Actually, $\theta_{0}$ is equal to a cone half-opening angle: $\theta_{0}=\theta$.

In (Nalewajko et al. 2009) it is proved that polarization angle is Lorentz invariant, i.e. does not transform in the observer and source reference frames, while for polarization degree (P.D.) $P$ it is not true. In case of synchrotron radiative mechanism in the optical thin jet with the helical magnetic field, the dependence is implemented (Lyutikov et al. 2005; Raiteri et al. 2013):

$$
P \sim P_{\text {max }} \sin ^{2} \xi^{\prime}
$$

where $P_{\max }$ is the highest observed P.D., $\xi^{\prime}$ is the angle between the observer and the instantaneous direction of the speed $v$ in the source frame. $\xi^{\prime}$ depends on $\xi$ in the observer frame by means of Lorentz transformations:

$$
\sin \xi^{\prime}=\frac{\sin \xi}{\Gamma(1-\beta \cos \xi)}=\delta \sin \xi
$$

where $\delta=(\Gamma(1-\beta \cos \xi))^{-1}$ is Doppler factor, $\Gamma=\left(1-\beta^{2}\right)^{-1 / 2}$ is Lorentz factor. In the observer frame $\xi$ could be determined:

$$
\cos \xi=\frac{(\dot{\rho} \cos \psi-\rho \dot{\psi} \sin \psi) \sin \zeta \sin i+\dot{z} \cos \zeta \cos i}{\sqrt{\dot{\rho}^{2}+\rho^{2} \dot{\psi}^{2}+\dot{z}^{2}}} .
$$

To calculate the model numerically for the object S5 $0716+714$ the jet kinematic parameters obtained majorly by radio observations were assumed. The inclination angle $i$ and half-opening angle of the cone $\theta_{0}$ were evaluated from radio observations by Pushkarev et al. (2009): $i=5^{\circ}, \theta_{0}=$ $1.5^{\circ}$. The value of maximum P.D. was measured by long monitoring (Larionov et al. 2013): $P_{\max }=0.3$. The physical speed of the optical jet components was confirmed to be equal $\beta>0.999 c$ (Butuzova 2018), that gave the Lorentz factor $\Gamma \approx 30$. A pitch-angle supposed to be small due to the model stability to its variations. The initial distance to the $z$-axis was appreciated as maximum:

$$
\rho_{0}=c \Delta t \approx 5 \cdot 10^{-5} p c,
$$

where $\Delta t \approx 1.5$ hour is the period of the flux and polarization variations obtained from our monitoring. The maximum value of angular momentum $j$ was used:

$$
j_{\text {max }}=\frac{L}{E_{\text {kin }}} c=\beta \rho_{0} .
$$

The result of the numerical model processing of the polarization vector motion is demonstrated on the $Q U$ diagram. In Fig. 7(a) the model of linear polarization variations during $\sim 40$ days is shown. The time is marked by colour. The absolute value of polarization - P.D. rises rapidly within the first several days (it corresponds to the "detwisting" on $Q U$-plane); then polarization begins to change slowly, the absolute value stays approximately constant. Eventually, the polarization vector produces a spiral on the $Q U$-plane.

Therefore, the jet geometry could cause the observed polarization vector variations on the $Q U$-diagram. Yet stable helical magnetic field structure could not be responsible 


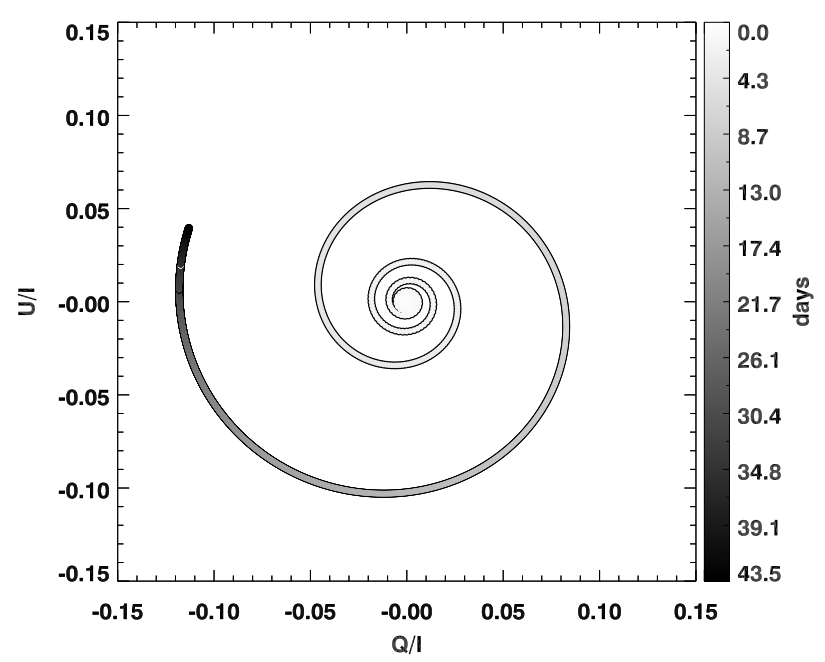

(a)

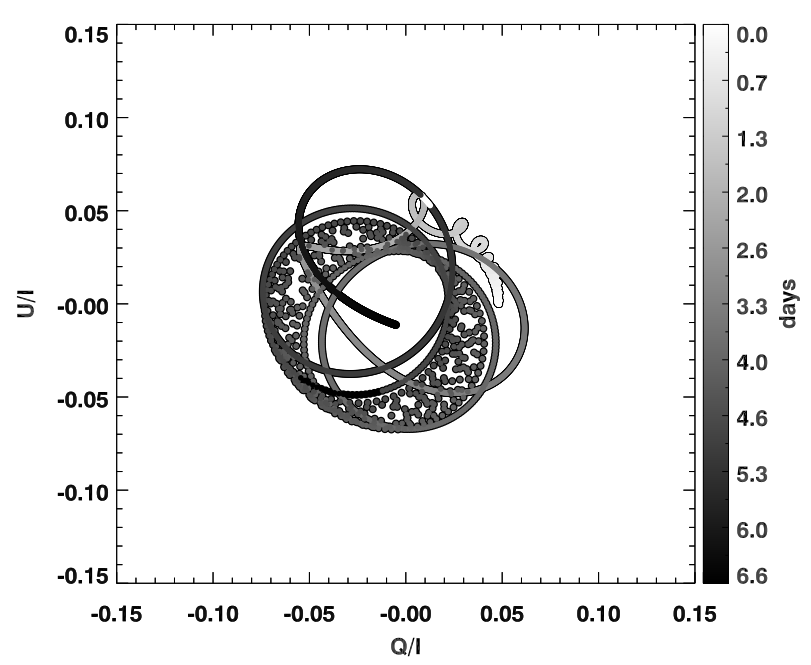

(b)

Figure 7. The results of the numerical model of polarization in jet: (a) without precession and (b) with precession.

for such complex trajectory: for instance, in (Impey et al. 2000) changing from night to night arches were recognized on the $Q U$-plane but without any evidence of common spiral production. The helical motion could be assumed to be burdened with an additional component. A precession appears as the most preferable kinematic effect since it is observationally confirmed for different types of AGNs, e.g. for Sy 1.5 Mrk 6 (e.g. Afanasiev et al. 2014). Here we consider precession is one of the possible reasons for the observed polarization variations.

The precession would be described as an additive value to the equations of the charged particle motion in the helical magnetic field. In this case the $\mathbf{E}_{\mathbf{p}}$ component is added to the electric vector value $\mathbf{E}$. $\mathbf{E}_{\mathbf{p}}$ is directed tangentially to the circle trajectory with the period of full cycle $T=2 \pi / \Omega$, the absolute value is constant. Then in the celestial frame $(u, w)$

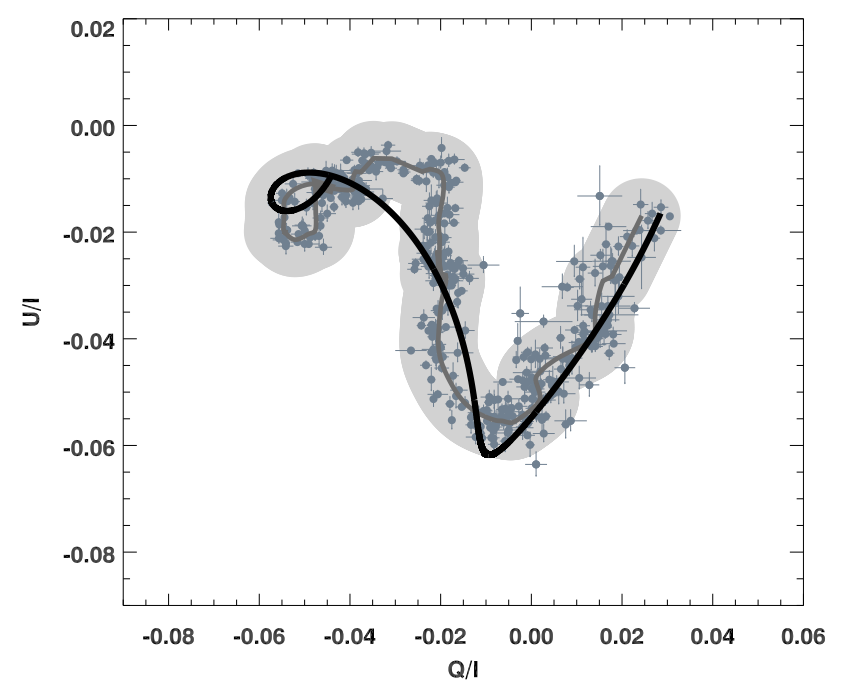

Figure 8. The model of linear polarization on the $Q U$-plane in case of jet precession. The observational data are plotted with grey dots with error bars. The $3 \sigma$ confidence area is coloured light grey.

Table 1. The parameters of numerical model of S5 $0716+714$ polarization with confirmed precession.

\begin{tabular}{lcc}
\hline Parameter & \multicolumn{1}{c}{ Value } \\
\hline Distance from the precession axis & $\omega$ & $0.7 \cdot 10^{-3} \mathrm{pc}$ \\
Precession period & $T$ & 15 days \\
\hline
\end{tabular}

$\mathbf{E}_{\mathbf{p}}$ would be equal to:

$$
\mathbf{E}_{\mathbf{p}}=\left(\frac{\sin \Omega}{\cos i}, \cos \Omega\right),
$$

consequentially, the electric vector position angle is determined:

$$
\tan \chi_{p}=\frac{\sin \Omega}{\cos i \cos \Omega} .
$$

To calculate the model with precession numerically we used parameters fitting best to our observational data. The selected parameters are presented in Tab. 1, the result of modelling could be found in Fig. 7(b). The model is significantly sensitive to the precession period $T$. It should be clarified that the observed precession motion of several active galaxies jets concerns the radio jet scales and average periods of years. Since we investigate much more compact regions $-10^{-3} \mathrm{pc}$, the much less period should be considered. Varying the model parameters we would like to fit average polarization variations particularly - both the form and the switch time. Finally, we have found out that the observed significant distortion of the particle motion could be explained only in case of small time-scales of the additive kinematic effect.

In Fig. 8 we present the result of fitting our data by the model of polarization in the helical magnetic field precessing with the period $\sim 15$ days. To testify to the goodness of our fit we have added a $3 \sigma$ confidence area of the smoothed po- 
larization vector rotation. One could discover that the model with the parameters described above assuredly fit the data with respect to the measurement errors. The exception is an interval of 21-22 hours, where the model curve falls out of the estimated area, yet we rely on the physical processes not considered in our geometrical model while it actually does not affect our quantitative outcomes.

\section{DISCUSSION}

The phenomenon of the S5 $0716+714$ intraday variability was suspected for a long time. However, to reveal the variations on the time-scale of hours the high accuracy and time resolution had been needed, that was not performed in longterm variability investigations. While the observations carried out by our group have met these demands.

One of the first investigations devoted to revealing of S5 0716+714 rapid variability was the work (Impey et al. 2000), where the monitoring of the object at the BTA in 1991 and 1994 was presented. The data plotted on $Q U$-plane circumscribed the apparent arches changing their positions from night to night. Due to the short sets (less than 2 hours), the unambiguous conclusions on the origin of changing electric vector direction could not be done.

In (Bhatta et al. 2015) the data of 16-hour polarimetric monitoring of S5 $0716+714$ was given. Comparing with (Impey et al. 2000) these observations have an order less time resolution $-\sim 20 \mathrm{~min}$ and the accuracy of polarization measurement $2-10 \%$. Though, the observation set was longer that discovered an approximately closed arch on the $Q U$-diagram.

One more investigation of the S5 0716+714 rapid variability was declared in (Sasada et al. 2008). It is essential that the data obtained by authors also perform an arch on the $Q U$-plane. Moreover, in this paper, the average time of flux variations was calculated as $\sim 15 \mathrm{~min}$. There the average time meant only the rising time of the bump, that agreed with the variability time-scale of $\sim 1.5$ hours, discovered by our observations, as we took into account not only the time of flux rising but the whole cycle of flux change. As a result, the assertion done in the paper that the size of the emitting region could be defined by 15 -minutes fluctuations seemed incorrect.

It should be mentioned that the variations of polarization vector along the arches on the $Q U$-plane has been registered at least for one more blazar - BL Lac. In (Covino et al. 2015) the 9-hour polarimetric monitoring of BL Lac was provided with the almost 1 min resolution and high accuracy of polarization measurement $-\sim 0.2-0.3 \%$. The monitoring was intermittently interrupted to observe the standard outside the field, but, nevertheless, the long dataset allowed to draw conclusions of variability pattern within the night. In the paper the polarization degree and angle were originally presented, yet we redrew the data on the $Q U$-plane (Fig. 9) revealing that the polarization vector were changing along the trajectory similar to S5 $0716+714$ one, and the time of the direction switch was close to the time calculated from our observations.

According to 9-hour monitoring, we are able to claim the intraday variability of flux and polarization of S5 $0716+714$. As $Q U$-plane reflects directly the plasma motion

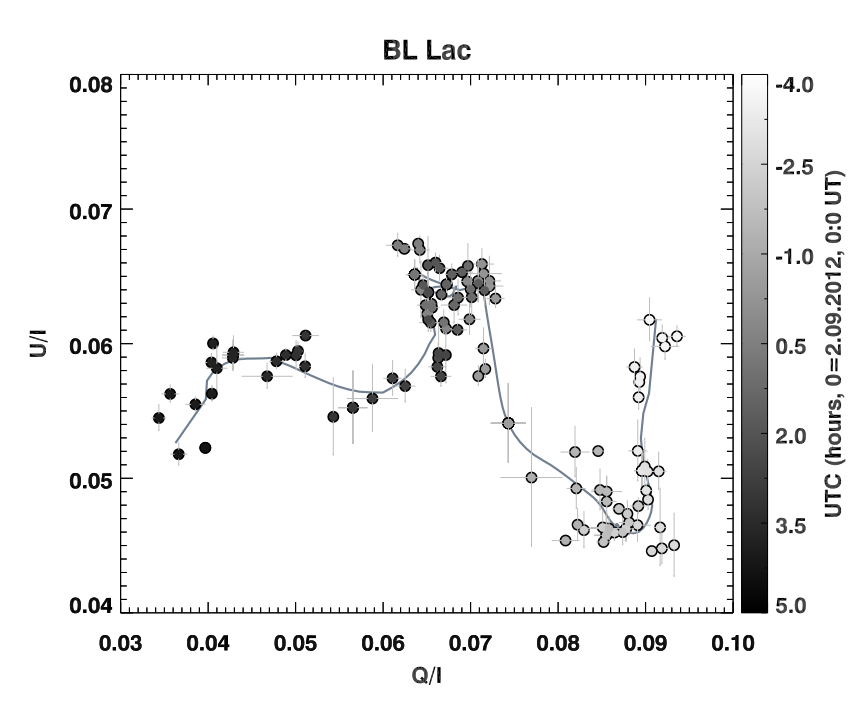

Figure 9. The variations of the Stokes parameters $Q$ and $U$ of BL Lac within 9 hours (Covino et al. 2015).

in the jet, the observed "arches" and "loops" bear out the common suggestions of the helical configuration of the magnetic field. Indeed, the ordered magnetic field with the helical structure in common produces the "arch"-like trajectories of charged particles radiating non-thermal polarized emission. Yet as it was shown above in case of absence of extra kinematic effects the polarization moves along the rings. Much more complicated paths on the $Q U$-plane could be explained with additional precession motion of either the magnetic field inside the jet or the outflow itself due to the precession of the central black hole. In the last case, the period agreed with observations seems too short, so the mechanism of such rapid jet "walk" is not clear. On the contrary, due to magnetohydrodynamics, the precession of the magnetic field on short time-scales is founded better.

To sum up, the observed phenomenon of rapid polarization variations could be qualitatively clarified only with geometrical effects. It is obvious that the submitted model is not absolutely accurate: it depends on a variety of parameters, not obtained for the optical jet, and also does not take into account the physical processes in plasma. Though, it could be distinctly declared that the geometrical effects dominate and highly accurately explain the variability.

Moreover, the time of polarization vector switch is very similar to the period of flux variations: $\sim 80 \mathrm{~min}$ for polarimetry and at least 1.5 hours for photometry. Comparing figures 3 and 5 one could see that even the dynamics of the variability development is the same: wavelet transform becomes inefficient when polarization begins to move along the arch without switching. Such behaviour adjusts with the earlier presented geometrical models (Butuzova 2018): while the emitting matter moves along the jet the Doppler factor varies that influences the boost of the relativistic plasma radiation. Therefore, the plasma motion in the jet affects not only the polarization vector rotation but the flux intensity as well that is in good agreement with the observations discovering the similarity of the total flux and polarization variations time-scale. 
The essential profit of our model of polarization in the jet is that as the polarization variation time - switch time and period of flux variations are equivalent they are closely connected to the linear scales of the emitting region. According to the common jet model (Gabuzda 2013), the plasma moves at the sides of the jet cone and, therefore, the time of polarization making the "loop" on the $Q U$-plane could be used to derive the size of the area: $r=c \Delta t$ and, in case of our results, $r \approx 1.5$ light hour or $5 \cdot 10^{-5} \mathrm{pc}$. This result is particularly important as the optical jet could not be resolved with modern techniques, and our estimation is the only possible indirect measurement of emitting area size.

\section{CONCLUSIONS}

In the given paper the intraday variability of S5 $0716+714$ was investigated and the following was done:

(i) during the 9-hour polarimetric monitoring carried out by the $6-m$ telescope the intraday variability of $S 50716+714$ was revealed in both total (with an amplitude $0.04 \mathrm{mag}$ ) and polarized $(\triangle P . D .=7 \%)$ light on the time-scale $\sim 1.5$ hour;

(ii) using $Q U$-plane to demonstrate the polarization vector variations we discovered the pattern of changes in polarized flux: within the night the polarization vector circumscribes "arches" and "loops". The similar scene was found for S5 0716+714 in several preceding papers (e.g. Impey et al. 2000; Bhatta et al. 2015), but the time resolution and accuracy were not valid enough. Such behaviour could not be revealed by plotting P.D. and polarization angle separately because of the vector quantity changes;

(iii) according to the general model of jet structure (Gabuzda 2013), the time of polarization vector variations is directly connected to the linear size of the emitting region: 1.5 light hour or $5 \cdot 10^{-5} \mathrm{pc}$ at the $\sim 10^{-3} \mathrm{pc}$ distance from the central black hole (optical jet). As optical jet is unresolved by modern techniques, we offered the results of indirect measurements based on polarimetric observations that are especially essential for jet physics researches;

(iv) we suggested a model of polarization producing by geometrical effects due to relativistic plasma motion in the helical magnetic field and, moreover, we added a feature, particularly new in numerical modelling, that only could qualitatively explain rapid polarization variability - the precession of the helical magnetic field;

(v) our model of relativistic plasma motion in the precessing magnetic field qualitatively and numerically describes our observational data; by fitting the model to the data, we calculated the period of precession - approximately 15 days. Furthermore, our model is able to reconduct the pattern of polarization vector motion on $Q U$-plane for BL Lac monitoring (Covino et al. 2015) as well.

\section{ACKNOWLEDGEMENTS}

We sincerely thank V.R. Amirkhanyan for valuable discussions and useful remarks. The results of observations were obtained with the 6-m BTA telescope of the Special Astrophysical Observatory Academy of Sciences, operating with the financial support of the Ministry of Education and Science of Russian Federation.

\section{REFERENCES}

Afanasiev V. L., Amirkhanyan V. R., 2012, Astrophys. Bull., 67, 4

Afanasiev V. L., Popovic L. C., Shapovalova A. I. et al., 2014, MNRAS, 440, 1

Amirkhanyan V. R., 2006, Astron. Rep., 50, 4

Bhatta G., Goyal A., Ostrowski M. et al., 2015, ApJ, 809, 2

Biermann P., Duerbeck H., Eckart A. et al., 1981, ApJ, 247:L53L56

Butuzova M. S., 2018, Astron. Rep., 62, 2

Capetti A. et al., 1997, A\&A, 317, 637-645

Covino S., Baglio M. C., Foschini L. et al. 2015, A\&A, 578, A68

Gabuzda D. C., 2013, EPJ Web of Conferences, 61

Geyer E. H., Jockers K., Kiselev N. N. et al., 1996, Ap\&SS, 239, 2

Gorshkov A. G., Ipatov A. V., Konnikova V. K. et al, 2011, Astron. Rep., 55, 12

Gorshkov A. G., Ipatov A. V., Konnikova V. K. et al., 2011, Astron. Rep., 55, 2

Gupta A.C., Wiita P. J., Pandey J. C. et al., 2009, preprint (arXiv:0906.3077)

Impey C. D., Bychkov V., Tapia S. et al., 2000, ApJ, 119, 4

Larionov V. M. et al., 2013, ApJ, 768, 40

Li H. Z., Jiang Y. G., Yi T. F. et al., 2018, Ap\&SS, 363, 3, 45

Liu X., Yang P. P., Liu J. et al., 2017, MNRAS, 469, 2

Lyutikov M., Pariev V. I., Gabuzda D. C., 2005, MNRAS, 360, 869-891

Meier D. L., Koide S., Uchida Y., 2001, Science, 291, 84

Nalewajko K., 2009, MNRAS, 395, 1

Nilsson K., Pursimo T., Sillanpaa A. et al., 2008, A\&A, 487, L29

Oliva E., 1996, A\&AS, 123, 589-592

Pushkarev A. B., Kovalev Y. Y., Lister M. L., Savolainen T., 2009, A\&A, 507, 33L

Raiteri C. M., Villata M., D'Ammando F. at WEBT, 2013, MNRAS, 436, 2

Rastorgueva E. A., Wiik K. J., Bajkova A. T. et al. 2011, A\&A, 529, A2

Sasada M., Uemura M., Arai A. et al., 2008, PASJ, 60, L37-L41.

Steffan W., Zensus J. A., Krichbaum T. P., 1995, A\&A, 302, 335

Zhang H., Li H., Guo F. et al., 2017, ApJ, 835, 2

Zhang X., Wu J., Meng N., 2018, MNRAS, 478, 3

Urry C. M., Scarpa R., O'Dowd M. et al., 2000, ApJ, 532, 2

This paper has been typeset from a $\mathrm{T}_{\mathrm{E}} \mathrm{X} / \mathrm{IAT}_{\mathrm{E}} \mathrm{X}$ file prepared by the author. 